\title{
Über die Stimmgabeln.
}

\section{Vierte Mitteilung. \\ Über die Geräusch in den Anfangsstadien der \\ Stimmgabelschwingungen.}

Von

Dr. H. Tsukamoto.

(Mit I Figur).

Aus der oto-rhino-laryngologischen Klinik (Direktor: Prof. Dr. T. HosHrNo)

and der III. medizinischen Klinik (Direktor: Prof. Dr. T. MASHIMO) der Kaiserlichen Universität zu Kioto.

\section{EINLEITUNG.}

Ich habe in vorstehender Mitteilung mittelst subjektiver Methode mit Königschen Stimmgabeln nachgewiesen, dass auch die mit Laufgewichten versehenen Hartmannschen Stimmgabeln in den Anfangsstadien ihrer Klangdauer im allgemeinen in grösserer oder geringerer Anzabl harmonische Obertöne produzieren, obgleich dem Hörer die Oktave wegen ihrer Verschmelzung mit dem Grundton nicht zum Bewusstsein zu kommen pflegt. Das ist der Fall, selbst wenn die Oktave in Wirklichkeit stark ist, solang der Grundton vorhanden ist. Beim Versuch zum Nachweis des Vorhandenseins der Oktave an den Hartmannschen, mit Laufgewichten beschwerten Stimmgabeln muss man dem Befestigungsgrad der Laufgewichte an den Gabelzinken besondere Aufmersamkeit schenken, weil man beim Erregen der belasteten Stimmgabeln mittelst verschiedener Methoden in den Anfangsstadien ihrer Klangdauer deutliche Geräusche hören kann, worüber noch keine Nachforschıngen angestellt worden sind. Ohne Berücksichtigung dieser Tatsache ist es sehr gewagt, über das Vorhandensein der Oktave an den Hartmannschen, mit Laufgewichten versehenen Stimmgabeln zu debattieren.

Ich will daher in dieser Mitteilung über einige von mir angestellte 
Beobachtungen über die Geräusche berichten, um über das Wesen der letzteren aufzuklären.

Dabei will ich die mit Laufgewichten versehenen Stimmgabeln, die in den Anfangsstadien ihrer Klangdauer deutliche Geräusche aufweisen, kurz als „Gabeln mit Geräusch” und die sonstigen Gabeln als ,Geräuschlose Gabeln" bezeichen.

\section{Erster Abschnitt.}

Häufigkeit der Stimmgabeln mit Geräusch.

1) Material für meine Experimente.

Ich wählte als Experimentiermaterial deri c'-Gabeln, drei c-Gabeln und zwei A-Gabeln, die alle aus Deutschland stammten, und zwolf c'-Gabeln, elf c-Gabeln und elf A-Gabeln aus Japan. Genauer angegeben, stammten drei c'-Gabeln, zwei c-Gabeln und zwei A-Gabeln aus der Dosaka, zwei $c^{\prime}$-Gabeln, zwei c-Gabeln sowie zwei A-Gabeln aus der Nakamura, zwei $\mathrm{c}^{\prime}$-Gabeln, zwei c-Gabeln und zwei A-Gabeln aus der Miyake, zwei c'Gabeln, zwei c-Gabeln und zwei A-Gabeln aus der Nagashima und endlich drei c'-Gabeln, drei c-Gabeln und drei A-Gabeln aus der Shiraimatsu. Alle diese neu gekauften, nicht verrosteten Stimmgabeln entsprechen den Gabeln, die wir täglich in der Klinik der Kaiserlichen Universität zu Kioto zu gebrauchen pflegen.

2) Messung der Häufigkeit der Stimmgabeln mit Geräusch mittelst subjektiver Methode.

Nicht alle der der Prüfung unterworfenen Stimmgabeln mit Laufgewichten zeigen hörbare Geräusche in den Anfangsstadien ihrer Klangdauer.

Daher habe ich die Stimmgabeln mit Geräusch nach dem Gehöre ausgewählt. Die folgende Tabelle bringt die Untersuchungsergebnisse.

\begin{tabular}{|c|c|c|c|c|}
\hline Fabrik & Nummer & C & C & C \\
\cline { 2 - 5 } & Geräusch & Geräusch & Geräusch \\
\hline Deutschland & 1 & $(-)$ & $(-)$ & $(-)$ \\
Deutschland & 2 & $(-)$ & $(-)$ & $(-)$ \\
Deutschland & 3 & $(-)$ & $(-)$ & \\
\hline
\end{tabular}




\begin{tabular}{|c|c|c|c|c|}
\hline Fabrik & Nummer & C' & C & A \\
\hline Dosaka & Geräusch & Geräusch & Geräusch \\
\hline Dosaka & 1 & $(+)$ & $(-)$ & $(+)$ \\
Dosaka & 3 & $(+)$ & $(+)$ & $(+)$ \\
\hline Nakamura & $(-)$ & & $(-)$ & $(-)$ \\
Nakamura & 2 & $(-)$ & $(+)$ & $(+)$ \\
\hline Mivake & 1 & $(+)$ & $(+)$ & $(-)$ \\
Mivake & 2 & $(+)$ & $(-)$ & $(+)$ \\
\hline Nagashima & 1 & $(-)$ & $(-)$ & $(-)$ \\
Nagashima & 2 & $(-)$ & $(-)$ & $(-)$ \\
\hline Schiraimatsu & 1 & $(+)$ & $(-)$ & $(+)$ \\
Schiraimatsu & 2 & $(-)$ & $(+)$ & $(-)$ \\
Schiraimatsu & 3 & $(-)$ & $(-)$ & $(+)$ \\
\hline
\end{tabular}

\section{Kritisches.}

1) Tch konnte deutliche Geräusche unter $15 \mathrm{c}^{\prime}$-Gabeln an sechs (40\%) nachweisen. Genauer angegeben, war an den Gabeln aus Deutschland und aus der Nagashima kein Geräusch zu konstatieren, während die erste und zweite $\mathrm{c}^{\prime}$-Gabel aus der Dosaka, die erste $\mathbf{c}^{\prime}$-Gabel aus der Nakamura, die erste und zweite $\mathrm{c}^{\prime}$-Gabel aus der Miyake und die erste $\mathrm{c}^{\prime}$-Gabel aus der Shiraimatsu hörbare metallisch klingende Geräusche aufwiesen.

2) Ferner konnte ich mit blossem Ohre ähnliche Geräusche unter vierzehn c-Gabeln an vier (28\%) feststellen, und zwar fand sich kein Geräusch an den Gabeln aus Deutschland oder aus der Nagashima, während die zweite c-Gabel aus der Dosaka, die zweite c-Gabel aus der Nakamura, die erste c-Gabel aus der Miyake und die zweite c-Gabel aus der Shiraimatsu deutliche Geräusche zeigten.

3) Schliesslich konnte ich deutliche Geräusche an fünf Gabeln unter dreizehn A-Gabeln (26\%) nachweisen. Auch hier wieder wiesen die Gabeln aus Deutschland oder aus der Nagashima kein Geräusch auf. Dagegen 
liessen die erste und zweite A-Gabel aus der Dosaka, die zweite A-Gabel aus der Nakamura, die zweite A-Gabel aus der Miyake und die erste A-Gabel aus der Shiraimatsu Gerausche erkennen.

\section{Zweiter Abschnitt. \\ Zeitdauer der Geräusche in den Anfangsstadien der Stimmgabelschwingungen. \\ Methodik des Experiments.}

Zur Bestimmung der Zeitdauer der Geräusche, welche wir in den Anfangsstadien der Stinumgabeltöne hören, benutzte ich die spezielle von Dr. J. Yoshioka konstruierte elektroakustische Einrichtung, die aus einem elektromagnetischen Mikrophon, mehreren Verstärkungseinrichtungen und einem Oszillographen besteht. Diese Einrichtung findet sich bereits in der ersten Mitteilung geschildert.

Weil bei dem Ausklingen einer Stimmgabel der Ton höher wird, (diese Tatsache wird spater noch genauer beschrieben werden) musste ich unbedingt eine möglichst konstante Anschlagskraft benutzen. Zu diesem Zwecke bediente ich mich einer bestimmten momentanen Kraft.

Ich liess nämlich die bestimmte Masse eines Perkussionshammers aus einer Lage bis zu einer auderen fallen, wobei ein Punkt des Hammergriffstiels als Drehpunkt wirkte.

Es wurde also potentielle Energie in einen bestimmten Betrag kinetischer Energie verwandelt.

Ferner registrierte ich die Geräusche der Stimmgabelu in den Anfangsstadien ihrer Klangdauer photographisch. Bei diesem Experimente verzichtete ich auf die mathematische Analyse der mittelst dieser experimentalen Einrichtung registrierten Gesäuschkurven, weil diese Einrichtung gegen die verschiedenen höheren Töne unter den Geräuschen unempfindlich war.

\section{Untersuchungsergebnisse.}

Ich habe die Untersuchungsergebnisse in der folgenden Tabelle angeordnet.

Bemerkungen :- 1 v.d. $=1$ Hertz $=2$.v.d̄. 


\begin{tabular}{|c|c|c|c|c|c|c|c|}
\hline \multirow[b]{2}{*}{ Fabrik } & \multirow[b]{2}{*}{ Nummer } & \multicolumn{2}{|c|}{$\mathrm{C}^{\prime}$} & \multicolumn{2}{|c|}{$\mathrm{C}$} & \multicolumn{2}{|c|}{$\mathbf{A}$} \\
\hline & & $\begin{array}{c}\text { Zeitdauer } \\
\text { des } \\
\text { Geräusches }\end{array}$ & $\begin{array}{l}\text { Schwin- } \\
\text { gungszahl }\end{array}$ & $\begin{array}{c}\text { Zeitdauer } \\
\text { des } \\
\text { Geräusches }\end{array}$ & \begin{tabular}{|c|} 
Schwin- \\
gungszahl
\end{tabular} & $\begin{array}{c}\text { Zeitdauer } \\
\text { des } \\
\text { Geräusches }\end{array}$ & $\begin{array}{l}\text { Schwin- } \\
\text { gungszahl }\end{array}$ \\
\hline Dosaka & 1 & $0.185^{\prime \prime}$ & 285v.d. & & & $0.865^{\prime \prime}$ & 111.1v.d. \\
\hline Dosaka & 2 & $0.355^{\prime \prime}$ & 211v.d. & $0.075^{\prime \prime}$ & 177.7 v.d. & $0.255^{\prime \prime}$ & 121.0 v.d. \\
\hline Nakamura & 1 & $0.13^{\prime \prime}$ & 200 v.d. & & & & \\
\hline Nakamura & 2 & & & $0.408^{\prime \prime} *$ & 177.7 v.d.* & $0.075^{\prime \prime}$ & 108.2v.d. \\
\hline Miyake & 1 & $0.122^{\prime \prime}$ & 259 v.d. & $0.868^{\prime \prime}$ & 152.8v.d. & & \\
\hline Miyake & 2 & $0.041^{\prime \prime}$ & 155.5ซ.d. & & & $0.133^{\prime \prime}$ & $98.2 v . d$ \\
\hline Schiraimatsu & 1 & $0.545^{\prime \prime}$ & 205.5v.d. & & & $0.038^{\prime \prime}$ & 97.7v.d. \\
\hline Schirairnatsu & 2 & & & $0.019^{\prime \prime}$ & 125.0v.d. & & \\
\hline
\end{tabular}

\section{Kritisches.}

Die Zeitdauer der Geräusche der belasteten $\mathrm{c}^{\prime}$-Gabeln beträgt 0,041 bis 0,545 Sekunde, die der c-Gabeln sowie der A-Gabeln je 0,019 bis 0,868 Sekunde und 0.038 bis 0,865 Sekunde.

\section{Dritter Abschnitt. \\ Über die Ursache der Geräusche.}

1) Über die Missverhältnisse zwischən der Zinkenbreite und der Breite des Belastungsraums.

Wir können mit den Fingern die Belastungen der Stimmgabeln mit Geräusch sehr leicht die Zinkenlänge entlang nach den Zinkenenden verschieben. Daher studierte die Befestigung der Stimmabellaufgewichte an den Gabelzinken.

Jede Belastung besitzt einen Hohlraum in Gestalt eines Parallelepipedons, durch den die Zinken passieren können. Die 6 Flächen dieses Parallelepipedons gehen den entsprechenden Zinkenflächen parallel.

Doch sind die Breite sowie die Tiefe des Hohlraums ein wenig grösser als die entsprechenden Grössen der Zinke. So ist hier stets ein Sprungfeder 
notwendig, um die Belastung gegen die Zinke zu pressen. Die Sprungfeder wirkt aber nur im Sinne der Tiefe, nicht aber in dem der Breite. Daher ist anzunehmen, dass die lockere Befestigung der Belastungen an die Gabelzinken auf dem Missverhältnis zwischen der Breite des Belastungsraums und der Zinkenbreite oder auf der Schwäche der Sprungfeder beruht. Diese Annahme liess mich erstens die Zinkenbreite mit der Breite des Belastungsraums vergleichen. Die dabei gewonnenen Untersuchungsergebnisse finden sich in der folgenden Tabelle angeordnet.

\begin{tabular}{|c|c|c|c|c|c|c|c|}
\hline \multirow[b]{2}{*}{ Fabrik } & \multirow[b]{2}{*}{ Nummer } & \multicolumn{2}{|c|}{$\mathrm{C}^{\prime}$} & \multicolumn{2}{|c|}{$\mathrm{C}$} & \multicolumn{2}{|c|}{$\mathbf{A}$} \\
\hline & & $\begin{array}{l}\text { Zinken- } \\
\text { breite }\end{array}$ & $\begin{array}{c}\text { Breite } d . \\
\text { Belas- } \\
\text { tungs- } \\
\text { raums } \\
\end{array}$ & $\begin{array}{c}\text { Zinken- } \\
\text { breite }\end{array}$ & $\begin{array}{c}\text { Breite d. } \\
\text { Belas- } \\
\text { tungs- } \\
\text { raums } \\
\end{array}$ & $\begin{array}{c}\text { Zinken- } \\
\text { breite }\end{array}$ & $\begin{array}{l}\text { Breite d. } \\
\text { Belas- } \\
\text { tungs- } \\
\text { raums } \\
\end{array}$ \\
\hline \multirow{6}{*}{ Dosaka } & 1 & 4.1 & 4.3 & 5.0 & 5.2 & 5.1 & 5.4 \\
\hline & 1 & 4.2 & 4.3 & 5.1 & 5.4 & 5.1 & 5.5 \\
\hline & 2 & 4.2 & 4.4 & 5.1 & 5.3 & 5.1 & 5.4 \\
\hline & 2 & 4.2 & 4.5 & 5.1 & 5.4 & 5.1 & 5.4 \\
\hline & 3 & 3.9 & 4.3 & & & & \\
\hline & 3 & 4.0 & 4.3 & & & & \\
\hline \multirow{6}{*}{ Deutschland } & 1 & 4.2 & 4.4 & 5.1 & 5.6 & 5.1 & 5.4 \\
\hline & 1 & 4.1 & 4.4 & 5.1 & 5.5 & 5.1 & 5.5 \\
\hline & 2 & 4.1 & 4.4 & 5.1 & 5.6 & 5.0 & 5.4 \\
\hline & 2 & 4.1 & 4.4 & 5.1 & 5.6 & 5.1 & 5.4 \\
\hline & 3 & 4.2 & 4.4 & 5.1 & 5.6 & & \\
\hline & 3 & 4.2 & 4.5 & 51 & 5.5 & & \\
\hline \multirow{4}{*}{ Nagashima } & 1 & 4.2 & 4.6 & 5.2 & 5.4 & 6.2 & 6.4 \\
\hline & 1 & 4.2 & 4.6 & 5.2 & 5.4 & 6.1 & 6.6 \\
\hline & 2 & 4.2 & 4.6 & 5.2 & 5.5 & 6.2 & 6.4 \\
\hline & 2 & 4.2 & 4.7 & 5.2 & 5.5 & 6.1 & 6.5 \\
\hline \multirow{4}{*}{ Shiraimatsu } & 1 & 3.9 & 4.2 & 5.1 & 5.3 & 5.1 & 5.4 \\
\hline & 1 & 3.9 & 4.2 & 5.2 & 5.4 & 5.1 & 5.3 \\
\hline & 2 & 3.9 & 4.1 & 5.0 & 5.3 & 5.0 & 5.3 \\
\hline & 2 & 4.0 & 4.2 & 5.1 & 5.3 & 5.1 & 5.3 \\
\hline
\end{tabular}




\begin{tabular}{|c|c|c|c|c|c|c|c|}
\hline \multirow{2}{*}{ Fabrik } & Nummer & \multicolumn{2}{|c|}{$\mathrm{C}^{\prime}$} & \multicolumn{2}{c|}{$\mathrm{C}$} & \multicolumn{2}{c|}{$\mathrm{A}$} \\
\cline { 2 - 8 } Shiraimatsu & Zinken- & $\begin{array}{c}\text { Breite d. } \\
\text { Bela-s } \\
\text { tungs- } \\
\text { raums }\end{array}$ & $\begin{array}{c}\text { Zinken- } \\
\text { breite }\end{array}$ & $\begin{array}{c}\text { Breite d. } \\
\text { Belas- } \\
\text { tungs- } \\
\text { raums }\end{array}$ & $\begin{array}{c}\text { Zinken- } \\
\text { breite }\end{array}$ & $\begin{array}{c}\text { Breite d. } \\
\text { Belas- } \\
\text { tungs- } \\
\text { raums }\end{array}$ \\
\hline \multirow{5}{*}{ Nakamura } & 3 & 4.0 & 4.3 & 5.1 & 5.4 & 5.1 & 5.4 \\
& 1 & 4.0 & 4.3 & 5.1 & 5.4 & 5.2 & 5.5 \\
& 2 & 4.9 & 4.3 & 5.0 & 5.3 & 5.2 & 5.4 \\
& 2 & 4.0 & 4.3 & 5.0 & 5.4 & 5.2 & 5.4 \\
& 2 & 4.0 & 4.3 & 5.0 & 5.5 & 5.2 & 5.4 \\
\hline \multirow{5}{*}{ Miyake } & 1 & 4.2 & 4.4 & 4.9 & 5.3 & 5.1 & 5.4 \\
& 1 & 4.2 & 4.4 & 5.0 & 5.3 & 5.1 & 5.4 \\
& 2 & 4.1 & 4.4 & 4.9 & 5.2 & 5.0 & 5.3 \\
& 2 & 4.2 & 4.3 & 4.9 & 5.2 & 5.0 & 5.3 \\
\hline
\end{tabular}

Aus der Tabelle geht hervor, dass der Grad des Missverhältnisses zwischen der Zinkenbreite und der Breite des Belastungsraums bei den geräuschlosen Stimmgabeln fast derselbe wie bei denen mit Geräusch ist.

2) Über die Reibungswiderstände zwischen den Belastungen sowie Sprungfedern und den Grabelzinken.

Da die lockere Befestigung der Laufgewichte gegen die Zinken wahrscheinlich von der Schwäche der Sprungfeder herrühren könnte, habe ich die Grösse des Reibungswiderstandes zwischen der Belastung sowie Sprungfeder und den Zinken gemessen.

a) Methodik der Messung der Reibungswiderstandsgrösse zwischen der Belastung sozie Sprungfeder und den Zinken.

Ich liess eine an der Belastung befestigte Schnur der horizontal gelegten Stimmgabel, deren breitere Zinkenfläche horizontal war, über eine Rolle gehen, befestigte an ihrem freien Ende eine Wagschale, deren Gewicht $48 \mathrm{~g}$ betrug, und legte auf diese solange Gewichte, bis dadurch die Belastung sowie die Sprungfeder eben noch in Bewegung gesetzt wurden oder; 
wenn angestossen, sich gleichförmig weiter bewegten.

\section{b) Untersuchungsergebnisse.}

Ich habe die verschiedene Werte der Reibungswiderstände unter Berücksichtigung des Gewichts der Wagschale im c.g. s-System in folgender Tabelle wiedergegeben.

\begin{tabular}{|c|c|c|c|c|c|c|c|}
\hline \multirow{2}{*}{ Fabsik } & \multirow{2}{*}{ Nummer } & \multicolumn{2}{|c|}{$\mathrm{C}^{\prime}$} & \multicolumn{2}{|c|}{$\mathrm{C}$} & \multicolumn{2}{|c|}{ A } \\
\hline & & g & $\mathrm{g}$ & g & g & $\mathrm{g}$ & $g$ \\
\hline \multirow{3}{*}{ Deutschland } & 1 & 6442.6 & 5882.1 & 4992.9 & 5722.3 & 8722.2 & 6772.6 \\
\hline & 2 & 7533.3 & 7677.1 & 5223.3 & 5772.9 & 7503.0 & 6777.0 \\
\hline & 3 & 5582.2 & 5943.2 & 5223.0 & 6013.4 & & \\
\hline \multirow{3}{*}{ Dosaka } & 1 & 783.5 & 3982.0 & 4803.0 & 5233.3 & 933.0 & 244.5 \\
\hline & 2 & 552.5 & 1220.4 & 2881.1 & 2903.3 & 2890.4 & 649.0 \\
\hline & 3 & 5592.4 & 6774.5 & & & & \\
\hline \multirow{2}{*}{ Nakamura } & 1 & 1889.0 & 1533.3 & 5999.0 & 6124.2 & 4789.0 & 5229.5 \\
\hline & 2 & 6332.4 & 5534.4 & 433.3 & 1339.9 & 2098.0 & 2692.2 \\
\hline \multirow{2}{*}{ Miyake } & 1 & 1993.0 & 4022.2 & 220.9 & $839.5^{\circ}$ & 5225.0 & 5722.2 \\
\hline & 2 & 2838.2 & 4630.4 & 5932.0 & 6098.0 & 990.2 & 2517.9 \\
\hline \multirow{2}{*}{ Nagashima } & 1 & 6732.5 & 5844.2 & 7233.0 & 5822.0 & 5933.5 & 6773.0 \\
\hline & 2 & 5522.5 & 5985.5 & 6633.0 & 6722.0 & 6235.5 & 6992.4 \\
\hline \multirow{3}{*}{ Shiraimatsu } & 1 & 255.1 & 287.0 & 5982.0 & 5772.2 & 4612.0 & 2912.4 \\
\hline & 2 & 6322.0 & 5972.4 & 2992.0 & 6335.5 & 5335.6 & 5452.3 \\
\hline & 3 & 6233.2 & 5982.4 & 6032.5 & 5998.0 & 6022.0 & 7032.9 \\
\hline
\end{tabular}

\section{Kritisches.}

Die Werte des Reibungswiderstandes zwischen den Belastungen sowie Sprungfedern und den Gabelzinken scheinen bei den Stimmgabeln mit Geräusçh viel kleiner als dịe bei den geräuschlosen zu sein. Es zeigte 
nämlich der Reibungswiderstand zwischen der Belastung sowie Sprungfeder und einer Zinke bei einer Stimmgabel mit Geräusch verschiedene Werte zwischen dem Maximalwert 2992,0g und dem Minimalwert 220,9g, während er bei den geräuschlosen Gabeln verschiedene Werte über $4789,0 \mathrm{~g}$ aufwies. Was das Verhältnis zwischen Zeitdauer der Geriusche und der Grösse des Reibungswiderstandes anbetrifft, so scheint nach der Tabelle zu gelten, dass die Zeitdauer der Geräusche um so länger ausfällt, je kleiner der Reibungswiderstand ist.

\section{Schluss.}

1) Ich konnte deutliche Geräusch unter zwei und vierzig belasteten Gabeln an $15(35,7 \%)$ in den Anfangsstadien ihrer Schwingungen konstatieren.

2) Die Zejtdauer der Geräusche schwankte zwischen 0,019 und 0,868 Sekunde.

3) Diese Geräusche sind nicht auf den Grade des Missverhältnisses zwischen der Zinkenbreite und der Breite des Belastungsraums zurückzuführen, sondern auf die Grösse des Reibungswiderstandes zwischen den Belastungen sowie Sprungfedern und den Zinkenoberflächen. Das Geräusch tritt auf, wenn der Reibungswiderstand kleiner als 2992,0g ist. 